\title{
ON THE LIMIT CYCLES FOR A CLASS OF FOURTH-ORDER DIFFERENTIAL EQUATIONS
}

\author{
JAUME LLIBRE $^{1}$ AND AMAR MAKHLOUF ${ }^{2}$
}

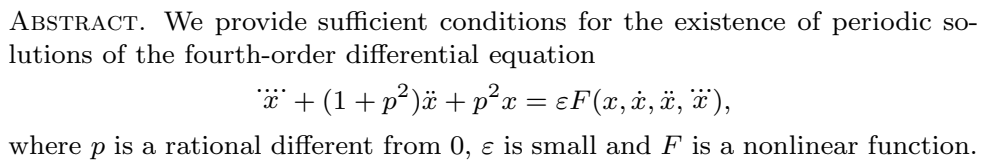

\section{INTRODUCTION AND STATEMENT OF THE MAIN RESULTS}

The objective of this paper is to study the periodic solutions of the fourth-order differential equation

$$
\dddot{x}+\left(1+p^{2}\right) \ddot{x}+p^{2} x=\varepsilon F(x, \dot{x}, \ddot{x}, \dddot{x}),
$$

where $p$ is a rational different from $0, \varepsilon$ is a small real parameter, and $F$ is a nonlinear function. The dot denotes derivative with respect to an independent variable $t$

Equations (1) appear in many places. For instance, these equations are contained in the differential equations (2) studied by Champneys [8], who analyzes these equations looking mainly for homoclinic orbits.

When $F(u, \dot{u}, \ddot{u}, \dddot{u})= \pm u^{2}$ equation (1) can come from the description of the travelling-wave solutions of the Korteweg-de Vries equation with an additional fifth-order dispersive term. Extended fifth-order Korteweg-de Vries equations have been considered in $[6,9,10,16,17,23]$. This equation has been used to describe chains of coupled nonlinear oscillators [25] and most notably gravity-capillary shallow water waves $[4,14,30]$. For other derivation of $(1)$ see $[12,13]$.

Another nonlinearity is $F(u, \dot{u}, \ddot{u}, \dddot{u})= \pm u^{3}$, then equation (1) is called the Extended Fischer-Kolmogorov equation or the Swift-Hohenberg equation see [5, 15], and in other places, see for instance the book $[26]$ and $[2,7]$.

In all these previous quoted references and for the corresponding differential equations (1) there studied, there are no proofs showing the existence of periodic orbits. Here for the differential equations (1) we provide two analytical algorithms for studying their periodic orbits, see Theorems 1 and 4 . Moreover, we shall illustrate the use of these algorithms in Corollaries 2 and 5.

In general to obtain analytically periodic solutions is a very difficult task, usually impossible. Here with the averaging theory we reduce this difficult problem for the differential equations (1) to find the zeros of a nonlinear system of three equations with three unknowns. We must say that the averaging theory for finding periodic

2010 Mathematics Subject Classification. 37G15, 37C80, 37C30.

Key words and phrases. periodic orbit, fourth-order differential equation, averaging theory. 
solutions in general does not provide all the periodic solutions of the system. For more information about the averaging theory see section 2 and the references quoted there.

The coefficients $1+p^{2}$ and $p^{2}$ in the differential equation (1) are quite special, but equation (1) with coefficients $p$ and $q$ instead of $1+p^{2}$ and $p^{2}$ already has been studied in the references $[19,21]$. But in those references is not studied the cases in which the unperturbed differential system has a double pair of imaginary eigenvalues, as it is the case for our unperturbed differential equation (1) which has eigenvalues $\pm i$ and $\pm p i$. Additionally since $p$ is rational this implies that the set of periodic orbits of the unperturbed differential equation (1) has dimension 4 . This is the case that we want to study in this paper.

We recall that a simple zero $r_{0}^{*}$ of a real function $\mathcal{F}\left(r_{0}\right)$ is defined by $\mathcal{F}\left(r_{0}^{*}\right)=0$ and $\left(d \mathcal{F} / d r_{0}\right)\left(r_{0}^{*}\right) \neq 0$.

Our first main result on the periodic solutions of this fourth-order differential equation (1) are the following.

Theorem 1. Let $p=p_{1} / p_{2}$ be a rational different from $-1,0,1$ with $p_{1}$ and $p_{2}$ coprime. For every $\left(r_{0}^{*}, Z_{0}^{*}, V_{0}^{*}\right)$ solution of the system

$$
F_{1}\left(r_{0}, Z_{0}, V_{0}\right)=0, \quad F_{2}\left(r_{0}, Z_{0}, V_{0}\right)=0, \quad F_{3}\left(r_{0}, Z_{0}, V_{0}\right)=0,
$$

satisfying

$$
\operatorname{det}\left(\left.\frac{\partial\left(F_{1}, F_{2}, F_{3}\right)}{\partial\left(r_{0}, Z_{0}, V_{0}\right)}\right|_{\left(r_{0}, Z_{0}, V_{0}\right)=\left(r_{0}^{*}, Z_{0}^{*}, V_{0}^{*}\right)}\right) \neq 0
$$

where

$$
\begin{aligned}
F_{1}\left(r_{0}, Z_{0}, V_{0}\right) & =\int_{0}^{\frac{2 \pi}{p}} \cos \theta F(\mathcal{A}, \mathcal{B}, \mathcal{C}, \mathcal{D}) d \theta \\
F_{2}\left(r_{0}, Z_{0}, V_{0}\right) & =-\int_{0}^{\frac{2 \pi}{p}} \frac{p^{2} V_{0} \sin \theta+r_{0} \sin (p \theta)}{p r_{0}} F(\mathcal{A}, \mathcal{B}, \mathcal{C}, \mathcal{D}) d \theta \\
F_{3}\left(r_{0}, Z_{0}, V_{0}\right) & =\int_{0}^{\frac{2 \pi}{p}} \frac{p^{2} Z_{0} \sin \theta-r_{0} \cos (p \theta)}{p r_{0}} F(\mathcal{A}, \mathcal{B}, \mathcal{C}, \mathcal{D}) d \theta
\end{aligned}
$$

with

$$
\begin{aligned}
\mathcal{A} & =\frac{r_{0} \sin \theta+V_{0} \sin (p \theta)-Z_{0} \cos (p \theta)}{p^{2}-1}, \\
\mathcal{B} & =\frac{r_{0} \cos \theta+p V_{0} \cos (p \theta)+p Z_{0} \sin (p \theta)}{p^{2}-1}, \\
\mathcal{C} & =\frac{p^{2}\left(Z_{0} \cos (p \theta)-V_{0} \sin (p \theta)\right)-r_{0} \sin \theta}{p^{2}-1}, \\
\mathcal{D} & =\frac{p^{3}\left(V_{0} \cos (p \theta)+Z_{0} \sin (p \theta)\right)+r_{0} \cos \theta}{1-p^{2}},
\end{aligned}
$$

the differential equation (1) has a periodic solution $x(t, \varepsilon)$ tending to the solution

$$
x(t)=\frac{r_{0}^{*} \sin t+V_{0}^{*} \sin (p t)-Z_{0}^{*} \cos (p t)}{p^{2}-1},
$$


of the equation

$$
\dddot{x}+\left(1+p^{2}\right) \ddot{x}+p^{2} x=0,
$$

when $\varepsilon \rightarrow 0$. Note that the period of $x(t)$ is the maximum of $2 \pi$ and $2 \pi p_{2}$.

Theorem 1 is proved in section 3 .

We shall provide two applications of Theorem 1. The first one is given in the following corollary.

Corollary 2. If $F(x, \dot{x}, \ddot{x}, \dddot{x})=\alpha \dot{x}-\beta \dot{x}^{3}$ with $\beta \neq 0$, then the differential equation (1) with $p=2$ has periodic solutions $x(t, \varepsilon)$ tending when $\varepsilon \rightarrow 0$ to the periodic solutions $x(t)$ of period $2 \pi$ of the equation $\dddot{x}+5 \ddot{x}+4 x=0$ given by (4) with the values $\left(r_{0}^{*}, Z_{0}^{*}, V_{0}^{*}\right)$ for which the determinant (3) is nonzero, where $r_{0}^{*}$ is a positive root of the polynomial $h_{11}\left(r_{0}\right) h_{12}\left(r_{0}\right) h_{13}\left(r_{0}\right)$ given by

$$
\begin{aligned}
h_{11}\left(r_{0}\right)= & \left(-480128+33075 \pi^{2}\right) \beta r_{0}^{2}+1208064 \alpha, \\
h_{12}\left(r_{0}\right)= & \left(-246290604621824+73505518387200 \pi^{2}-7346142720000 \pi^{4}+\right. \\
& \left.246140015625 \pi^{6}\right) \beta^{4} r_{0}^{8}-180\left(-30786325577728+8900719411200 \pi^{2}-\right. \\
& \left.855187200000 \pi^{4}+27348890625 \pi^{6}\right) \alpha \beta^{3} r_{0}^{6}+32400(-962072674304+ \\
& \left.281705971712 \pi^{2}-27027302400 \pi^{4}+850854375 \pi^{6}\right) \alpha^{2} \beta^{2} r_{0}^{4}- \\
& 1512000\left(-34359738368+10377658368 \pi^{2}-1003544640 \pi^{4}+\right. \\
& \left.31255875 \pi^{6}\right) \alpha^{3} \beta r_{0}^{2}+3292047360000 \pi^{2}(-8+3 \pi)(8+3 \pi) \alpha^{4}, \\
& \left(7(-256+105 \pi)(256+105 \pi) \cdot\left(524288+33075 \pi^{2}\right)^{2} \beta^{6} r_{0}^{12}-\right. \\
& 108\left(524288+33075 \pi^{2}\right) \cdot\left(164014063616-8795852800 \pi^{2}+\right. \\
& \left.310629375 \pi^{4}\right) \alpha \beta^{5} r_{0}^{10}+2592\left(51272426226450432+42303540428800 \pi^{2}-\right. \\
& \left.106125626880000 \pi^{4}+2148407296875 \pi^{6}\right) \alpha^{2} \beta^{4} r_{0}^{8}- \\
& 155520\left(3705079307698176-16934442106880 \pi^{2}-6389957700000 \pi^{4}+\right. \\
& \left.85693190625 \pi^{6}\right) \alpha^{3} \beta^{3} r_{0}^{6}+518400(1540553229467648- \\
& \left.18794559504384 \pi^{2}-2087128814400 \pi^{4}+29536801875 \pi^{6}\right) \alpha^{4} \beta^{2} r_{0}^{4}- \\
& 217728000\left(-137438953472+11055808512 \pi^{2}-1108054080 \pi^{4}+\right. \\
& \left.\left.31255875 \pi^{6}\right) \alpha^{5} \beta r_{0}^{2}+474054819840000 \pi^{2}(-16+3 \pi)(16+3 \pi) \alpha^{6}\right),
\end{aligned}
$$

and for every one of these $r_{0}^{*}$ we must obtain the solutions $\left(Z_{0}^{*}, V_{0}^{*}\right)$ of the system

$$
\begin{aligned}
& -105 \pi \beta r_{0}^{3}-1344 Z_{0} \beta r_{0}^{2}+1260 \pi \alpha r_{0}-840 \pi V_{0}^{2} \beta r_{0}-840 \pi Z_{0}^{2} \beta r_{0}+ \\
& 6720 Z_{0} \alpha-2048 Z_{0}^{3} \beta-2816 V_{0}^{2} Z_{0} \beta=0, \\
& 56 \beta r_{0}^{4}+105 \pi Z_{0} \beta r_{0}^{3}-840 \alpha r_{0}^{2}+576 V_{0}^{2} \beta r_{0}^{2}+768 Z_{0}^{2} \beta r_{0}^{2}-630 \pi Z_{0} \alpha r_{0}+ \\
& 210 \pi Z_{0}^{3} \beta r_{0}+210 \pi V_{0}^{2} Z_{0} \beta r_{0}+3360 V_{0}^{2} \alpha-1152 V_{0}^{4} \beta-1024 V_{0}^{2} Z_{0}^{2} \beta=0 .
\end{aligned}
$$

In particular if $\alpha=\beta=1$ then the differential equation (1) with $p=2$ has six periodic solutions corresponding to the values of $\left(r_{0}^{*}, Z_{0}^{*}, V_{0}^{*}\right)$ given by

$$
\begin{aligned}
& (0.1471021308,-1.5590893313,0.8704857802), \\
& (0.1471021308,-1.5590893313,-0.8704857802), \\
& (1.7006853293,-2.3247555702,0), \\
& (2.2708080828,0.8053695810,0), \\
& (3.8007389635,-2.9941595054,0), \\
& (3.9910593631,-1.5239474038,0) .
\end{aligned}
$$

The stability of these periodic solutions is studied at the end of the proof of this corollary. 
The second application of Theorem 1 is on a generalization of the Korteweg-de Vries equation, because instead of taking $F(u, \dot{u}, \ddot{u}, \dddot{u})= \pm u^{2}$ we take $F(u, \dot{u}, \ddot{u}, \dddot{u})=$ $a u+b \dot{u}+c \ddot{u}+d \ddot{u}+e u^{2}$.

Corollary 3. If $F(u, \dot{u}, \ddot{u}, \dddot{u})=a u+b \dot{u}+c \ddot{u}+d \dddot{u}+e u^{2}$, then the differential equation (1) with $p=2$ has periodic solutions $x(t, \varepsilon)$ tending when $\varepsilon \rightarrow 0$ to the periodic solutions $x(t)$ of period $2 \pi$ of the equation $\dddot{x}+5 \ddot{x}+4 x=0$ given by (4) with $\left(r_{0}^{*}, Z_{0}^{*}, V_{0}^{*}\right)$ solutions of the nonlinear system

$$
\begin{aligned}
F_{1}\left(r_{0}, Z_{0}, V_{0}\right)= & \frac{1}{270 \pi}\left(15 e \pi V_{0} r_{0}+45 b \pi r_{0}-45 d \pi r_{0}+120 a V_{0}-480 c V_{0}+\right. \\
& \left.240 b Z_{0}-960 d Z_{0}-16 e V_{0} Z_{0}\right)=0 \\
F_{2}\left(r_{0}, Z_{0}, V_{0}\right)= & -\frac{1}{540 \pi r_{0}}\left(128 e V_{0}^{3}-480 b V_{0}^{2}+1920 d V_{0}^{2}+224 e r_{0}^{2} V_{0}+\right. \\
& 112 e Z_{0}^{2} V_{0}+225 a \pi r_{0} V_{0}-360 c \pi r_{0} V_{0}+240 a Z_{0} V_{0}- \\
& 960 c Z_{0} V_{0}+60 e \pi r_{0} Z_{0} V_{0}+120 b r_{0}^{2}-120 d r_{0}^{2}+90 b \pi r_{0} Z_{0}- \\
& \left.360 d \pi r_{0} Z_{0}\right)=0, \\
F_{3}\left(r_{0}, Z_{0}, V_{0}\right)= & \frac{1}{1080 \pi r_{0}}\left(15 e \pi r_{0}^{3}+120 a r_{0}^{2}-120 c r_{0}^{2}+432 e Z_{0} r_{0}^{2}+120 e \pi Z_{0}^{2} r_{0}-\right. \\
& 180 b \pi V_{0} r_{0}+720 d \pi V_{0} r_{0}+450 a \pi Z_{0} r_{0}-720 c \pi Z_{0} r_{0}+224 e Z_{0}^{3}+ \\
& \left.480 a Z_{0}^{2}-1920 c Z_{0}^{2}+256 e V_{0}^{2} Z_{0}-960 b V_{0} Z_{0}+3840 d V_{0} Z_{0}\right)=0,
\end{aligned}
$$

for which the determinant (3) is nonzero. In particular, if we take $a=1, b=-1$, $c=2, d=-2$ and $e=1$, we have a periodic solution corresponding to the values

$$
\left(r_{0}^{*}, Z_{0}^{*}, V_{0}^{*}\right)=(8.69721625612798 . .,-0.57977009742156 . ., 0.607127989348911 . .) \text {, }
$$

whose determinant $(3)$ is equal to $0.1379945424519633 . . \neq 0$. This periodic solution has a 2-dimensional stable invariant manifold and 1-dimensional unstable invariant manifold.

Corollaries 2 and 3 are proved in section 5 .

We remark that the results of Theorem 1 when the rational $|p|>1$ have been also obtained using a different result from averaging theory in [19], but the results of Theorem 1 are new when $|p|<1$.

In the next result we shall study the fourth-order differential equation (1) with $p= \pm 1$, and we will allow that the equation be non autonomous.

Theorem 4. Assume that in the differential equation (1) we have $p= \pm 1$ and $F$ additionally depends on $t$, i.e $F\left(t, x, x^{\prime}, x^{\prime \prime}, x^{\prime \prime \prime}\right)$. If $\left(Z_{0}^{*}, V_{0}^{*}\right)$ is such that $f_{1}\left(Z_{0}^{*}, V_{0}^{*}\right)=$ $f_{2}\left(Z_{0}^{*}, V_{0}^{*}\right)=0$ and

where

$$
\operatorname{det}\left(\left.\frac{\partial\left(f_{1}, f_{2}\right)}{\partial\left(Z_{0}, V_{0}\right)}\right|_{\left(Z_{0}, V_{0}\right)=\left(Z_{0}^{*}, V_{0}^{*}\right)}\right) \neq 0,
$$

$$
\begin{aligned}
& f_{1}\left(Z_{0}, V_{0}\right)=-\int_{0}^{2 \pi} F(t, a, b, c, d) \sin t d t \\
& f_{2}\left(Z_{0}, V_{0}\right)=-\int_{0}^{2 \pi} F(t, a, b, c, d) \cos t d t
\end{aligned}
$$


with

$$
\begin{aligned}
& a=-\frac{1}{2}\left(Z_{0} \cos t+V_{0} \sin t\right), \\
& b=\frac{1}{2}\left(V_{0} \cos t+Z_{0} \sin t\right), \\
& c=\frac{1}{2}\left(Z_{0} \cos t-V_{0} \sin t\right), \\
& d=-\frac{1}{2}\left(V_{0} \cos t+Z_{0} \sin t\right),
\end{aligned}
$$

then for $\varepsilon \in\left[-\varepsilon_{0}, \varepsilon_{0}\right]$ with $\varepsilon_{0}$ sufficiently small, there is a $2 \pi$-periodic solution $x(t, \varepsilon)$ of equation (1) tending to the solution $x_{0}(t)$ of equation (1) with $\varepsilon=0$ given by

$$
x(t, \varepsilon) \rightarrow \frac{1}{2}\left(V_{0}^{*} \sin t-Z_{0}^{*} \cos t\right) \quad \text { as } \varepsilon \rightarrow 0 .
$$

Theorem 4 is proved in section 6. Again its proof is based in the averaging theory for computing periodic orbits. The averaging results used in the proofs of Theorems 1 and 4 are different. An application of Theorem 4 is given to the equation

$$
\dddot{x}+2 \ddot{x}+x=\varepsilon(b \sin t+\sin x),
$$

More precisely we have the next result.

Corollary 5. If $F(t, x, \dot{x}, \ddot{x}, \dddot{x})=b \sin t+\sin x$, then for every positive integer $m$ there exists an $\varepsilon_{0}>0$ such that for all $\varepsilon \in\left(0, \varepsilon_{0}\right)$ the differential equation (1) has at least $m$ periodic solutions.

Corollary 5 is proved in section 7 .

\section{BASIC RESUlts ON AVERAGING THEORY}

In this section we present the basic results from the averaging theory that we shall need for proving the main results of this paper.

We consider the problem of the bifurcation of $T$-periodic solutions from differential systems of the form

$$
\dot{\mathbf{x}}=F_{0}(t, \mathbf{x})+\varepsilon F_{1}(t, \mathbf{x})+\varepsilon^{2} F_{2}(t, \mathbf{x}, \varepsilon),
$$

with $\varepsilon=0$ to $\varepsilon \neq 0$ sufficiently small. Here the functions $F_{0}, F_{1}: \mathbb{R} \times \Omega \rightarrow \mathbb{R}^{n}$ and $F_{2}: \mathbb{R} \times \Omega \times\left(-\varepsilon_{0}, \varepsilon_{0}\right) \rightarrow \mathbb{R}^{n}$ are $\mathcal{C}^{2}$ functions, $T$-periodic in the first variable, and $\Omega$ is an open subset of $\mathbb{R}^{n}$. The main assumption is that the unperturbed system

$$
\dot{\mathbf{x}}=F_{0}(t, \mathbf{x}),
$$

has a submanifold of periodic solutions. A solution of this problem is given using the averaging theory.

Let $\mathbf{x}(t, \mathbf{z}, \varepsilon)$ be the solution of the system (8) such that $\mathbf{x}(0, \mathbf{z}, \varepsilon)=\mathbf{z}$. We write the linearization of the unperturbed system along a periodic solution $\mathbf{x}(t, \mathbf{z}, 0)$ as

$$
\dot{\mathbf{y}}=D_{\mathbf{x}} F_{0}(t, \mathbf{x}(t, \mathbf{z}, 0)) \mathbf{y} .
$$

In what follows we denote by $M_{\mathbf{z}}(t)$ some fundamental matrix of the linear differential system (9).

We assume that there exists an open set $V$ with $\mathrm{Cl}(V) \subset \Omega$ such that for each $\mathbf{z} \in \mathrm{Cl}(V), \mathbf{x}(t, \mathbf{z}, 0)$ is $T$-periodic, where $\mathbf{x}(t, \mathbf{z}, 0)$ denotes the solution of the unperturbed system (8) with $\mathbf{x}(0, \mathbf{z}, 0)=\mathbf{z}$. The set $\mathrm{Cl}(V)$ is isochronous for the 
system (7); i.e. it is a set formed only by periodic orbits, all of them having the same period. Then, an answer to the bifurcation problem of $T$-periodic solutions from the periodic solutions $\mathbf{x}(t, \mathbf{z}, 0)$ contained in $\mathrm{Cl}(V)$ is given in the following result.

Theorem 6. [Perturbations of an isochronous set] We assume that there exists an open and bounded set $V$ with $\mathrm{Cl}(V) \subset \Omega$ such that for each $\mathbf{z} \in \mathrm{Cl}(V)$, the solution $\mathbf{x}(t, \mathbf{z})$ is $T$-periodic, then we consider the function $\mathcal{F}: \mathrm{Cl}(V) \rightarrow \mathbb{R}^{n}$

$$
\mathcal{F}(\mathbf{z})=\int_{0}^{T} M_{\mathbf{z}}^{-1}(t, \mathbf{z}) F_{1}(t, \mathbf{x}(t, \mathbf{z})) d t .
$$

If there exists $a \in V$ with $\mathcal{F}(a)=0$ and $\operatorname{det}((d \mathcal{F} / d \mathbf{z})(a)) \neq 0$, then there exists a $T$-periodic solution $\varphi(t, \varepsilon)$ of system $(7)$ such that $\varphi(0, \varepsilon) \rightarrow a$ as $\varepsilon \rightarrow 0$.

For an easy proof of Theorem 6 see Corollary 1 of [3]. In fact the result of Theorem 6 is a classical result due to Malkin [22] and Roseau [27]

We denote by $\xi^{\perp}: \mathbb{R}^{k} \times \mathbb{R}^{n-k} \rightarrow \mathbb{R}^{n-k}$ the projection of $\mathbb{R}^{n}$ onto its last $n-k$ coordinates; i.e. $\xi^{\perp}\left(x_{1}, \ldots, x_{n}\right)=\left(x_{k+1}, \ldots, x_{n}\right)$.

We assume that there exists a k-dimensional submanifold $\mathcal{Z}$ of $\Omega$ filled with $\mathrm{T}$ periodic orbits of (8). Then an answer to the problem of bifurcation of T-periodic solutions from the periodic solutions contained in $\mathcal{Z}$ for system (8) is giving in the following result proved in [20].

Theorem 7. Assume $n=2 m$. Let $V$ be an open and bounded subset of $\mathbb{R}^{m}$, and let $\beta: C l(V) \rightarrow \mathbb{R}^{m}$ be a $\mathcal{C}^{2}$ function. We assume that

(a) $\mathcal{Z}=\left\{\mathbf{z}_{\alpha}=(\alpha, \beta(\alpha)), \alpha \in C l(V)\right\} \subset \Omega$ and for each $\mathbf{z}_{\alpha} \in \mathcal{Z}$ the solution $\mathbf{x}\left(\mathbf{t}, \mathbf{z}_{\alpha}\right)$ of (8) is T-periodic;

(b) for each $\mathbf{z}_{\alpha} \in \mathcal{Z}$ there is a fundamental matrix $M_{\mathbf{z}_{\alpha}}(t)$ of (9) such that the matrix $M_{\mathbf{z}_{\alpha}}^{-1}(0)-M_{\mathbf{z}_{\alpha}}^{-1}(T)$ has at the upper right corner a $m \times m$ matrix $\triangle_{\alpha}$ with $\operatorname{det}\left(\triangle_{\alpha}\right) \neq 0$ and at the lower right corner the $m \times m$ zero matrix.

We consider the function $F: C l(V) \rightarrow \mathbb{R}^{m}$

$$
F(\alpha)=\xi^{\perp}\left(\frac{1}{T} \int_{0}^{T} M_{\mathbf{z}_{\alpha}}^{-1}(t) F_{1}\left(t, x\left(t, \mathbf{z}_{\alpha}\right)\right) d t\right) .
$$

If there exists $a \in V$ with $F(a)=0$ and $\operatorname{det}\left(\left(\frac{d F}{d \alpha}\right)(a)\right) \neq 0$, then there is a $T$ - periodic solution $\varphi(t, \varepsilon) \rightarrow \mathbf{z}_{\alpha}$ as $\varepsilon \rightarrow 0$.

Theorem 7 was proved recently in [20], it extends the original results of Malkin and Roseau to another set of assumptions.

\section{Proof of Theorem 1}

Introducing the variables $(x, y, z, v)=(x, \dot{x}, \ddot{x}, \dddot{x})$ we write the fourth-order differential equation (1) as the following first-order differential system

$$
\begin{aligned}
& \dot{x}=y, \\
& \dot{y}=z, \\
& \dot{z}=v, \\
& \dot{v}=-p^{2} x-\left(1+p^{2}\right) z+\varepsilon F(x, y, z, v) .
\end{aligned}
$$


defined in an open subset $\Omega$ of $\mathbb{R}^{4}$. Of course as before the dot denotes derivative with respect to the independent variable $t$. System (12) with $\varepsilon=0$ will be called the unperturbed system, otherwise we have the perturbed system. The unperturbed system has a unique singular point at the origin with eigenvalues $\pm i$, and $\pm i p$. We shall write system (12) in such a way that the linear part at the origin will be in its real Jordan normal form. Then doing the change of variables $(x, y, z, v) \rightarrow$ $(X, Y, Z, V)$ given by

$$
\left(\begin{array}{c}
X \\
Y \\
Z \\
V
\end{array}\right)=\left(\begin{array}{cccc}
0 & p^{2} & 0 & 1 \\
p^{2} & 0 & 1 & 0 \\
1 & 0 & 1 & 0 \\
0 & -1 / p & 0 & -1 / p
\end{array}\right)\left(\begin{array}{l}
x \\
y \\
z \\
v
\end{array}\right)
$$

the differential system (12) becomes

$$
\begin{aligned}
\dot{X} & =-Y+\varepsilon G(X, Y, Z, V), \\
\dot{Y} & =X, \\
\dot{Z} & =-p V \\
\dot{V} & =p Z-\frac{\varepsilon}{p} G(X, Y, Z, V),
\end{aligned}
$$

where $G(X, Y, Z, V)=F(A, B, C, D)$ with

$$
A=\frac{Y-Z}{p^{2}-1}, \quad B=\frac{p V+X}{p^{2}-1}, \quad C=\frac{P^{2} Z-Y}{p^{2}-1}, \quad D=\frac{p^{3} V+X}{1-p^{2}} .
$$

Note that the linear part of the differential system (14) at the origin is in its real normal Jordan form and that the change of variables (11) is defined when $\mathrm{p}$ is different from $-1,0,1$, because the determinant of the matrix of the change is $\left(p^{2}-1\right)^{2} / p$.

We pass from the cartesian variables $(X, Y, Z, V)$ to the cylindrical ones $(r, \theta, Z, V)$ of $\mathbb{R}^{4}$, where $X=r \cos \theta$ and $Y=r \sin \theta$. In these new variables the differential system (14) can be written as

$$
\begin{aligned}
\dot{r} & =\varepsilon \cos \theta H(r, \theta, Z, V), \\
\dot{\theta} & =1-\varepsilon \frac{\sin \theta}{r} H(r, \theta, Z, V), \\
\dot{Z} & =-p V, \\
\dot{V} & =p Z-\frac{\varepsilon}{p} H(r, \theta, Z, V),
\end{aligned}
$$

where $H(r, \theta, Z, V)=F(a, b, c, d)$ with

$$
a=\frac{r \sin \theta-Z}{p^{2}-1}, \quad b=\frac{p V+r \cos \theta}{p^{2}-1}, \quad c=\frac{p^{2} Z-r \sin \theta}{p^{2}-1}, \quad d=\frac{p^{3} V+r \cos \theta}{1-p^{2}} .
$$


We change the independent variable from $t$ to $\theta$, and denoting the derivative with respect to $\theta$ by a prime the differential system (15) becomes

$$
\begin{aligned}
r^{\prime} & =\varepsilon \cos \theta H+O\left(\varepsilon^{2}\right), \\
Z^{\prime} & =-p V-\varepsilon \frac{p V \sin \theta}{r} H+O\left(\varepsilon^{2}\right), \\
V^{\prime} & =p Z+\varepsilon \frac{p^{2} Z \sin \theta-r}{p r} H+O\left(\varepsilon^{2}\right),
\end{aligned}
$$

where $H=H(r, \theta, Z, V)$.

We shall apply Theorem 6 to the differential system (16). We note that system (16) can be written as system (7) taking

$$
\mathbf{x}=\left(\begin{array}{c}
r \\
Z \\
V
\end{array}\right), \quad t=\theta, \quad F_{0}(\theta, \mathbf{x})=\left(\begin{array}{c}
0 \\
-p V \\
p Z
\end{array}\right)
$$

and

$$
F_{1}(\theta, \mathbf{x})=\left(\begin{array}{c}
\cos \theta H \\
-\frac{p V \sin \theta}{r} H \\
\frac{p^{2} Z \sin \theta-r}{p r} H
\end{array}\right) .
$$

We shall study the periodic solutions of system (8) in our case, i.e. the periodic solutions of system (16) with $\varepsilon=0$. These periodic solutions are

$$
\left(\begin{array}{c}
r(\theta) \\
Z(\theta) \\
V(\theta)
\end{array}\right)=\left(\begin{array}{c}
r_{0} \\
Z_{0} \cos (p \theta)-V_{0} \sin (p \theta) \\
V_{0} \cos (p \theta)+Z_{0} \sin (p \theta)
\end{array}\right)
$$

for $\left(r_{0}, Z_{0}, V_{0}\right)$ with $r_{0}>0$; This set of periodic orbits has dimension three, all having the same period $2 \pi / p$. To look for the periodic solutions of our equation (1) we must calculate the zeros $\alpha=\left(r_{0}, Z_{0}, V_{0}\right)$ of the system $\mathcal{F}(\alpha)=0$, where $\mathcal{F}(\alpha)$ is given (10). The fundamental matrix $M(\theta)$ of the differential system (16) with $\varepsilon=0$ along any periodic solution is

$$
M(\theta)=M_{\mathbf{z}_{\alpha}}(\theta)=\left(\begin{array}{ccc}
1 & 0 & 0 \\
0 & \cos (p \theta) & -\sin (p \theta) \\
0 & \sin (p \theta) & \cos (p \theta)
\end{array}\right) .
$$

Now computing the function $\mathcal{F}(\alpha)$ given in (10) we got that the system $\mathcal{F}(\alpha)=0$ can be written as

$$
\left(\begin{array}{c}
F_{1}(r, Z, V) \\
F_{2}(r, Z, V) \\
F_{3}(r, R, V)
\end{array}\right)=\left(\begin{array}{l}
0 \\
0 \\
0
\end{array}\right)
$$


where

$$
\begin{aligned}
F_{1}(r, Z, V) & =\int_{0}^{\frac{2 \pi}{p}} \cos \theta F(\mathcal{A}, \mathcal{B}, \mathcal{C}, \mathcal{D}) d \theta \\
F_{2}(r, Z, V) & =-\int_{0}^{\frac{2 \pi}{p}} \frac{p^{2} V_{0} \sin \theta+r_{0} \sin (p \theta)}{p r_{0}} F(\mathcal{A}, \mathcal{B}, \mathcal{C}, \mathcal{D}) d \theta \\
F_{3}(r, Z, V) & =\int_{0}^{\frac{2 \pi}{p}} \frac{p^{2} Z_{0} \sin \theta-r_{0} \cos (p \theta)}{p r_{0}} F(\mathcal{A}, \mathcal{B}, \mathcal{C}, \mathcal{D}) d \theta
\end{aligned}
$$

with $\mathcal{A}, \mathcal{B}, \mathcal{C}$ and $\mathcal{D}$ as in the statement of Theorem (1).

The zeros $\left(r^{*}, R^{*}, V^{*}\right)$ of system (17) with respect to the variables $r, Z$ and $V$ provide periodic orbits of system (17) with $\varepsilon \neq 0$ sufficiently small if they are simple , i.e. if the determinant (3) is nonzero. Going back through the change of variable, for every simple zero $\left(r^{*}, Z^{*}, V^{*}\right)$ of system (17), we obtain a $2 \pi p_{1}$ periodic solution $\mathrm{x}(\mathrm{t})$ of the differential equation (1) for $\varepsilon \neq 0$ sufficiently small such that $x(t)$ tends to the periodic solution

$$
x(t)=\frac{r_{0}^{*} \sin t+V_{0}^{*} \sin (p t)-Z_{0}^{*} \cos (p t)}{p^{2}-1},
$$

of equation

$$
\dddot{x}+\left(1+p^{2}\right) \ddot{x}+p^{2} x=0,
$$

when $\varepsilon \rightarrow 0$. This complete the proof of Theorem 1 .

\section{The Resultant of POlynomials}

Let the roots of the polynomial $P(x)$ with leading coefficient one be denoted by by $a_{i}, i=1,2, \ldots, n$ and those of the polynomial $Q(x)$ with leading coefficient one be denoted by $b_{j}, \mathrm{j}=1,2, \ldots, \mathrm{m}$. The resultant of $P$ and $Q, \operatorname{Res}[P, Q]$, is the expression formed by the product of all the differences $a_{i}-b_{j}, i=1,2, \ldots, n, j=1,2, \ldots, m$. In order to see how to compute $\operatorname{Res}[P, Q]$, see for instance [18] and [24]. The main property of the resultant is that if $P$ and $Q$ have a common solution, then necessarily $\operatorname{Res}[P, Q]=0$.

Consider now two polynomials $P(X, Y)$ and $Q(X, Y)$. These polynomials can be considered as polynomials in $X$ with polynomial coefficients in $Y$. Then the resultant with respect to $\mathrm{X}, \operatorname{Res}[P, Q, X]$, is a polynomial in the variable $\mathrm{Y}$ with the following property. If $P(X, Y)$ and $Q(X, Y)$ have a common solution $\left(X_{0}, Y_{0}\right)$ then $\operatorname{Res}[P, Q, X]\left(Y_{0}\right)=0$, and similarly for the variable $X$. In particular, if the polynomials depending on one variable,

$$
\begin{aligned}
p(X) & =\operatorname{Res}[P, Q, Y], \\
q(Y) & =\operatorname{Res}[P, Q, X],
\end{aligned}
$$

have finitely many solutions (i.e. they are not the zero polynomial), then the zero polynomial system

$$
P(X, Y)=0, \quad Q(X, Y)=0,
$$

has finitely many solutions. 
Finally consider three polynomials $P(X, Y, Z), Q(X, Y, Z)$ and $R(X, Y, Z)$. First these polynomials can be considered as polynomials in $\mathrm{X}$ with polynomial coefficients in $\mathrm{Y}$ and $\mathrm{Z}$. Then the resultants with respect to $\mathrm{X}, p q(Y, Z)=\operatorname{Res}[P, Q, X]$ and $\operatorname{pr}(Y, Z)=\operatorname{Res}[P, R, X]$, are polynomials in the variables $Y$ and $Z$ with the following property. If $P(X, Y, Z), Q(X, Y, Z)$ and $R(X, Y, Z)$ have a common solution $\left(X_{0}, Y_{0}, Z_{0}\right)$, then $p q\left(Y_{0}, Z_{0}\right)=0$ and $\operatorname{pr}\left(Y_{0}, Z_{0}\right)=0$. Now the resultant $p(Z)=\operatorname{Res}[p q, p r, Y]$ is a polynomial in $Z$ such that $p\left(Z_{0}\right)=0$. In a similar way we can construct polynomials $q(Y)$ and $r(X)$ such that $q\left(Y_{0}\right)=0$ and $r\left(X_{0}\right)=0$. If every of the polynomials $p(Z), q(Y)$ and $r(X)$ are non-zero, then the polynomial system

$$
P(X, Y, Z)=0, \quad Q(X, Y, Z)=0, \quad R(X, Y, Z)=0,
$$

has finitely many solutions.

\section{Proof of Corollaries 2 AND 3}

All the computations of this section has been checked with the help of the algebraic manipulators mathematica and maple.

Proof of Corollary 2. If $F(x, \dot{x}, \ddot{x}, \dddot{x} \dddot{x})=\alpha \dot{x}-\beta \dot{x}^{3}$, then the system (2) of the statement of Theorem 1 is

$$
\begin{aligned}
& F_{1}\left(r_{0}, Z_{0}, V_{0}\right)=\frac{f_{1}\left(r_{0}, Z_{0}, V_{0}\right)}{7560 \pi}=0, \\
& F_{2}\left(r_{0}, Z_{0}, V_{0}\right)=\frac{f_{2}\left(r_{0}, Z_{0}, V_{0}\right)}{3780 \pi r_{0}}=0, \\
& F_{3}\left(r_{0}, Z_{0}, V_{0}\right)=\frac{f_{3}\left(r_{0}, Z_{0}, V_{0}\right)}{3780 \pi r_{0}}=0,
\end{aligned}
$$

where

$$
\begin{aligned}
f_{1}\left(r_{0}, Z_{0}, V_{0}\right)= & -105 \pi \beta r_{0}^{3}-1344 Z_{0} \beta r_{0}^{2}+1260 \pi \alpha r_{0}-840 \pi V_{0}^{2} \beta r_{0}- \\
& 840 \pi Z_{0}^{2} \beta r_{0}+6720 Z_{0} \alpha-2048 Z_{0}^{3} \beta-2816 V_{0}^{2} Z_{0} \beta, \\
f_{2}\left(r_{0}, Z_{0}, V_{0}\right)= & 56 \beta r_{0}^{4}+105 \pi Z_{0} \beta r_{0}^{3}-840 \alpha r_{0}^{2}+576 V_{0}^{2} \beta r_{0}^{2}+768 Z_{0}^{2} \beta r_{0}^{2}- \\
& 630 \pi Z_{0} \alpha r_{0}+210 \pi Z_{0}^{3} \beta r_{0}+210 \pi V_{0}^{2} Z_{0} \beta r_{0}+3360 V_{0}^{2} \alpha- \\
& 1152 V_{0}^{4} \beta-1024 V_{0}^{2} Z_{0}^{2} \beta \\
f_{3}\left(r_{0}, Z_{0}, V_{0}\right)= & V_{0}\left(105 \pi \beta r_{0}^{3}+480 Z_{0} \beta r_{0}^{2}-630 \pi \alpha r_{0}+210 \pi V_{0}^{2} \beta r_{0}+\right. \\
& \left.210 \pi Z_{0}^{2} \beta r_{0}-3360 Z_{0} \alpha+1024 Z_{0}^{3} \beta+1152 V_{0}^{2} Z_{0} \beta\right) .
\end{aligned}
$$

To solve this system we use the method of the resultant explained in section 4 . First we compute the following two resultants

$$
\begin{aligned}
& g_{1}\left(r_{0}, Z_{0}\right)=\operatorname{Res}\left[f_{1}, f_{2}, V_{0}\right]=256 \beta^{2}\left(g_{11}\left(r_{0}, Z_{0}\right)\right)^{2}, \\
& \left.g_{2}\left(r_{0}, Z_{0}\right)=\operatorname{Res}\left[f_{1}, f_{3}, V_{0}\right]\right]=-4 \beta^{2} g_{21}\left(r_{0}, Z_{0}\right)\left(g_{22}\left(r_{0}, Z_{0}\right)\right)^{2},
\end{aligned}
$$


where

$$
\begin{aligned}
g_{11}\left(r_{0}, Z_{0}\right)= & -1499400 \pi^{2} \beta^{2} r_{0}^{6}+3472875 \pi^{3} Z_{0} \beta^{2} r_{0}^{5}-55050240 \pi Z_{0} \beta^{2} r_{0}^{5}+ \\
& 13759200 \pi^{2} Z_{0}^{2} \beta^{2} r_{0}^{4}-238551040 Z_{0}^{2} \beta^{2} r_{0}^{4}+1587600 \pi^{2} \alpha \beta r_{0}^{4}- \\
& 19998720 \pi Z_{0}^{3} \beta^{2} r_{0}^{3}-13891500 \pi^{3} Z_{0} \alpha \beta r_{0}^{3}+128862720 \pi Z_{0} \alpha \beta r_{0}^{3}+ \\
& 107956800 \pi^{2} \alpha^{2} r_{0}^{2}+2822400 \pi^{2} Z_{0}^{4} \beta^{2} r_{0}^{2}+18874368 Z_{0}^{4} \beta^{2} r_{0}^{2}- \\
& 129124800 \pi^{2} Z_{0}^{2} \alpha \beta r_{0}^{2}+770703360 Z_{0}^{2} \alpha \beta r_{0}^{2}+711244800 \pi Z_{0} \alpha^{2} r_{0}+ \\
& 42147840 \pi Z_{0}^{5} \beta^{2} r_{0}-325785600 \pi Z_{0}^{3} \alpha \beta r_{0}+722534400 Z_{0}^{2} \alpha^{2}+ \\
& 67108864 Z_{0}^{6} \beta^{2}-440401920 Z_{0}^{4} \alpha \beta \\
g_{21}\left(r_{0}, Z_{0}\right)= & 105 \pi \beta r_{0}^{3}+1344 Z_{0} \beta r_{0}^{2}-1260 \pi \alpha r_{0}+840 \pi Z_{0}^{2} \beta r_{0}-6720 Z_{0} \alpha+2048 Z_{0}^{3} \beta, \\
g_{22}\left(r_{0}, Z_{0}\right)= & 33075 \pi^{2} \beta r_{0}^{4}+147840 \pi Z_{0} \beta r_{0}^{3}-132300 \pi^{2} \alpha r_{0}^{2}-98304 Z_{0}^{2} \beta r_{0}^{2}- \\
& 866880 \pi Z_{0} \alpha r_{0}+26880 \pi Z_{0}^{3} \beta r_{0}-860160 Z_{0}^{2} \alpha+262144 Z_{0}^{4} \beta .
\end{aligned}
$$

Now we compute the resultant

$$
h_{1}\left(r_{0}\right)=\operatorname{Res}\left[g_{11}, g_{21} g_{22}, Z_{0}\right]=A \pi^{6} r_{0}^{14} \beta^{12}\left(h_{11}\left(r_{0}\right)\right)^{4} h_{12}\left(r_{0}\right) h_{13}\left(r_{0}\right),
$$

where $A$ is a negative integer and the polynomials $h_{11}\left(r_{0}\right), h_{12}\left(r_{0}\right)$ and $h_{13}\left(r_{0}\right)$ are defined in the statement of the corollary. Now, by Theorem 1, it follows the proof of the corollary for $\alpha$ and $\beta$ arbitrary.

In what follows we shall prove the rest of the corollary when $\alpha=\beta=1$. Let $h_{1}\left(r_{0}\right)=h_{11}\left(r_{0}\right) h_{12}\left(r_{0}\right) h_{13}\left(r_{0}\right)$. We have computed using the algebraic manipulator mathematica the 10 positive real roots $r_{0}$ of the polynomial $h_{1}\left(r_{0}\right)$ of degree 22 , obtaining

$$
\begin{array}{rlll}
r_{0}^{1}=0.1471021308, & r_{0}^{2}=1.6388943848, & r_{0}^{3}=1.7006853293, \\
r_{0}^{4}=2.2233102811, & r_{0}^{5}=2.2708080828, & r_{0}^{6}=2.6693890712, \\
r_{0}^{7}=2.8036318630, & r_{0}^{8}=3.8007389635, & r_{0}^{9}=3.9910593631, \\
r_{0}^{10}=5.5058622914 . & &
\end{array}
$$

From the properties of the resultant if system (2) has a solution $\left(r_{0}^{*}, Z_{0}^{*}, V_{0}^{*}\right)$, then $r_{0}^{*}$ must be one of the previous ten roots. So now we solve the system

$$
f_{1}\left(r_{0}^{k}, Z_{0}, V_{0}\right)=0, \quad f_{2}\left(r_{0}^{k}, Z_{0}, V_{0}\right)=0, \quad f_{3}\left(r_{0}^{k}, Z_{0}, V_{0}\right)=0,
$$

for $k=1, \ldots, 10$. The unique of such systems having solutions are for $k=$ $1,3,5,8,9$, the first has two solutions and the others only one. These solutions $\left(r_{0}^{*}, Z_{0}^{*}, V_{0}^{*}\right)$ are

$$
\begin{aligned}
& s_{1}=(0.1471021308,-1.5590893313,0.8704857802), \\
& s_{2}=(0.1471021308,-1.5590893313,-0.8704857802), \\
& s_{3}=(1.7006853293,-2.3247555702,0), \\
& s_{4}=(2.2708080828,0.8053695810,0), \\
& s_{5}=(3.8007389635,-2.9941595054,0), \\
& s_{6}=(3.9910593631,-1.5239474038,0) .
\end{aligned}
$$

For these six solutions of system (2) we have that every determinant

$$
\operatorname{det}\left(\left.\frac{\partial\left(F_{1}, F_{2}, F_{3}\right)}{\partial\left(r_{0}, Z_{0}, V_{0}\right)}\right|_{\left(r_{0}, Z_{0}, V_{0}\right)=\left(r_{0}^{*}, Z_{0}^{*}, V_{0}^{*}\right)}\right) \neq 0,
$$

is not zero. Therefore, from Theorem 1 Corollary 2 is proved. 
In the case of the periodic orbits of Corollary 2 if the eigenvalues of the Jacobian matrix

$$
\left(\left.\frac{\partial\left(F_{1}, F_{2}, F_{3}\right)}{\partial\left(r_{0}, Z_{0}, V_{0}\right)}\right|_{\left(r_{0}, Z_{0}, V_{0}\right)=\left(r_{0}^{*}, Z_{0}^{*}, V_{0}^{*}\right)}\right)
$$

have non-zero real part, then the kind of stability of the fixed point of the Poincaré return map corresponding to the periodic orbit associated to the initial conditions $\left(r_{0}^{*}, Z_{0}^{*}, V_{0}^{*}\right)$ is the same as the stability of the equilibrium point $\left(r_{0}^{*}, Z_{0}^{*}, V_{0}^{*}\right)$ of the averaged system

$$
\dot{r}=f_{1}(r, Z, V), \quad \dot{Z}=f_{2}(r, Z, V), \quad \dot{V}=f_{3}(r, Z, V) .
$$

For more details see for instance [11, 22, 27]. More precisely, the eigenvalues of the matrix (18) in the points $s_{k}$ are

$$
\begin{array}{ccc}
-0.230966 & 0.083853 & 1.31027 \\
-1.25208 & -0.118096+0.0795022 i & -0.118096-0.0795022 i \\
0.0239955 & -0.0654383+0.241454 i & -0.0654383-0.241454 i \\
-0.331964 & -0.016883 & 0.0216052 \\
-0.048336 & -0.0475268+0.149255 i & -0.0475268-0.149255 i \\
-0.0926494 & 0.0129701+0.160721 i & 0.0129701-0.160721 i
\end{array}
$$

for $k=1, \ldots, 6$ respectively. Therefore, the Poincaré return map associated to the periodic orbit corresponding to the solution $s_{1}$ or $s_{6}$ has a stable manifold of dimension 1 and an unstable one of dimension 2; the one corresponding to the solution $s_{3}$ or $s_{4}$ has a stable manifold of dimension 2 and an unstable one of dimension 1 ; and the one corresponding to the solution $s_{2}$ or $s_{5}$ has a stable manifold of dimension 3 (so this periodic orbit is a local atractor). This completes the proof of the corollary.

Proof of Corollary 3. If $F(u, \dot{u}, \ddot{u}, \dddot{u})=a u+b \dot{u}+c \ddot{u}+d \dddot{u}+e u^{2}$, then the system (2) of the statement of Theorem 1 becomes

$$
F_{1}\left(r_{0}, Z_{0}, V_{0}\right)=0, \quad F_{2}\left(r_{0}, Z_{0}, V_{0}\right)=0, \quad F_{3}\left(r_{0}, Z_{0}, V_{0}\right)=0
$$

where the functions $F_{k}$ for $k=1,2,3$ are given in the statement of Corollary 3 . We will compute the explicit solutions of this system for the values $a=1, b=-1$, $c=2, d=-2$ and $e=1$. To solve this system for these values we use the method of the resultant explained in section 4 . First we compute the following two resultants

$$
g_{1}\left(r_{0}, Z_{0}\right)=\operatorname{Res}\left[f_{1}, f_{2}, V_{0}\right], \quad g_{2}\left(r_{0}, Z_{0}\right)=\operatorname{Res}\left[f_{1}, f_{3}, V_{0}\right]
$$

After we calculate the resultant

$$
h\left(r_{0}\right)=\operatorname{Res}\left[g_{1}, g_{2}, Z_{0}\right]
$$


and we obtain

$$
\begin{aligned}
h\left(r_{0}\right)= & -\frac{A}{r_{0}^{3}}\left(9 \pi r_{0}-490\right)^{6}\left(1130234765625 \pi^{11} r_{0}^{6}-13783764330000 \pi^{9} r_{0}^{6}-\right. \\
& 60381440409600 \pi^{7} r_{0}^{6}-129029610405888 \pi^{5} r_{0}^{6}-249593166036992 \pi^{3} r_{0}^{6}- \\
& 200111116255232 \pi r_{0}^{6}-301709389275000 \pi^{10} r_{0}^{5}+ \\
& 3664818815616000 \pi^{8} r_{0}^{5}+2825329036492800 \pi^{6} r_{0}^{5}- \\
& 13354791988101120 \pi^{4} r_{0}^{5}-58485745416929280 \pi^{2} r_{0}^{5}+ \\
& 8488229766430720 r_{0}^{5}+629450345671875 \pi^{11} r_{0}^{4}+ \\
& 57047220004320000 \pi^{9} r_{0}^{4}-1059288983182080000 \pi^{7} r_{0}^{4}+ \\
& 4383568900915200000 \pi^{5} r_{0}^{4}-5994037617308467200 \pi^{3} r_{0}^{4}+ \\
& 2574524515300147200 \pi r_{0}^{4}-100511542618200000 \pi^{10} r_{0}^{3}- \\
& 4451720423270400000 \pi^{8} r_{0}^{3}+71341344366501888000 \pi^{6} r_{0}^{3}- \\
& 206696066303655936000 \pi^{4} r_{0}^{3}+234734116254253056000 \pi^{2} r_{0}^{3}+ \\
& 12150737358290944000 r_{0}^{3}+20158184726391600000 \pi^{9} r_{0}^{2}- \\
& 117206575334392320000 \pi^{7} r_{0}^{2}-259007665408450560000 \pi^{5} r_{0}^{2}- \\
& 1896854203284848640000 \pi^{3} r_{0}^{2}+1016965008489185280000 \pi r_{0}^{2}- \\
& 590545792140595200000 \pi^{8} r_{0}+598111246178611200000 \pi^{6} r_{0}+ \\
& 22142776638740889600000 \pi^{4} r_{0}+1103169498749337600000 \pi^{2} r_{0}+ \\
& 20618861617368000000000 \pi^{7}-157135609541836800000000 \pi^{5}+ \\
& \left.110167062714777600000000 \pi^{3}\right)
\end{aligned}
$$

where $A$ is a positive constant. This polynomial only provides the following two positive real roots for $r_{0}$ :

$$
\text { 8.69721625612798.. and } 17.33020491445082 .
$$

Now we must substitute these values of $r_{0}$ into the system (19) and obtain the solutions for the variables $\left(Z_{0}, V_{0}\right)$. Only the first of these two roots provides solutions for $\left(Z_{0}, V_{0}\right)$, which are

$$
Z_{0}=-0.57977009742156 . ., \quad V_{0}=0.607127989348911 . .
$$

Since the determinant (3) on this solution is nonzero, by Theorem 1 we obtain a periodic solution for the corresponding differential equation.

The eigenvalues of the Jacobian matrix which appears in (3) are

$$
\text { 1.426999265250410..,-1.035682690159468.., -0.0933708781987750. . }
$$

So the periodic solution that we find has a stable invariant manifold of dimension 2 and an unstable invariant manifold of dimension 1.

\section{Proof of Theorem 4}

We want to study the periodic orbits of the class of fourth-order differential equation

$$
\dddot{x}+2 \ddot{x}+x=\varepsilon F(t, x, \dot{x}, \ddot{x}, \dddot{x}),
$$

This is the case of equation (1) when $p= \pm 1$. Note that now we are including the possibility that the differential equation be non-autonomous. 
Introducing the variables $(x, y, z, v)=(x, \dot{x}, \ddot{x}, \dddot{x})$ we write the fourth-order differential equation (20) as the following first-order differential system

$$
\begin{aligned}
& \dot{x}=y, \\
& \dot{y}=z, \\
& \dot{z}=v, \\
& \dot{v}=-x-2 z+\varepsilon F(t, x, y, z, v) .
\end{aligned}
$$

defined in an open subset $\Omega$ of $\mathbb{R}^{4}$. Of course as before the dot denotes derivative with respect to the independent variable $t$. System $(21)$ with $\varepsilon=0$ will be called the unperturbed system, otherwise we have the perturbed system. The unperturbed system has a unique singular point at the origin with eigenvalues $i, i,-i,-i$. We shall write system (21) in such a way that the linear part at the origin will be in its real Jordan normal form. Then doing the change of variables $(x, y, z, v) \rightarrow(X, Y, Z, V)$ given by

$$
\left(\begin{array}{l}
X \\
Y \\
Z \\
V
\end{array}\right)=\left(\begin{array}{cccc}
0 & 1 & 0 & 1 \\
1 & 0 & 1 & 0 \\
0 & 0 & 2 & 0 \\
0 & 1 & 0 & -1
\end{array}\right)\left(\begin{array}{l}
x \\
y \\
z \\
v
\end{array}\right)
$$

the differential system (21) becomes

$$
\begin{aligned}
& \dot{X}=-Y+\varepsilon G(t, X, Y, Z, V), \\
& \dot{Y}=X, \\
& \dot{Z}=X-V, \\
& \dot{V}=Y+Z-\varepsilon G(t, X, Y, Z, V),
\end{aligned}
$$

where $G(t, X, Y, Z, V)=F(t, A, B, C, D)$ with

$$
A=Y-\frac{Z}{2}, \quad B=\frac{X+V}{2}, \quad C=\frac{Z}{2}, \quad D=\frac{X-V}{2} .
$$

Note that the linear part of the differential system (23) at the origin is in its real normal Jordan form. We shall apply Theorem 7 to the differential system (23). We note that this system (23) can be written as system (7) taking

$$
\mathbf{x}=\left(\begin{array}{c}
X \\
Y \\
Z \\
V
\end{array}\right), \quad \mathbf{F}_{\mathbf{0}}(t, \mathbf{x})=\left(\begin{array}{c}
-Y \\
X \\
X-V \\
Y+Z
\end{array}\right)
$$

and

$$
\mathbf{F}_{\mathbf{1}}(t, \mathbf{x})=\left(\begin{array}{c}
G(t, X, Y, Z, V) \\
0 \\
0 \\
-G(t, X, Y, Z, V)
\end{array}\right) .
$$

We shall study the periodic solutions of system (8) in our case, i.e. the periodic solutions of the unperturbed system (23). Clearly these periodic solutions are

$$
\mathbf{x}(t)=\left(\begin{array}{c}
X(t) \\
Y(t) \\
Z(t) \\
V(t)
\end{array}\right)=\left(\begin{array}{c}
0 \\
0 \\
Z_{0} \cos t-V_{0} \sin t \\
V_{0} \cos t+Z_{0} \sin t
\end{array}\right)
$$


These periodic solutions are circles on the plane $X=Y=0$ of the unperturbed system (23). All theses periodic orbits have period $2 \pi$ in the variable $t$. These periodic orbits filled a plane minus the origin. We shall describe the different elements which appear in the statement of Theorem 7 in the particular case of the differential system (23). Thus we have that $k=2$ and $n=4$. We take the open bounded subset $V$ of $\mathbb{R}^{2}$ as $V=\left\{\left(Z_{0}, V_{0}\right): Z_{0}^{2}+V_{0}^{2}<r^{2}\right\}$, with $r>0$ fixed. Here $\alpha=\left(Z_{0}, V_{0}\right)$, and $\beta: V \rightarrow \mathbb{R}^{2}$ defined by $\beta\left(Z_{0}, V_{0}\right)=(0,0)$. The set $\mathcal{Z}$ is

$$
\mathcal{Z}=\left\{\mathbf{z}_{\alpha}=\left(Z_{0}, V_{0}, 0,0\right):\left(Z_{0}, V_{0}\right) \in V\right\} .
$$

So from now in order to apply Theorem 7 we need to write system (23) as follows,

$$
\begin{aligned}
& \dot{Z}=X-V, \\
& \dot{V}=Y+Z-\varepsilon G(t, X, Y, Z, V), \\
& \dot{X}=-Y+\varepsilon G(t, X, Y, Z, V), \\
& \dot{Y}=X,
\end{aligned}
$$

Computing the fundamental matrix $M_{\mathbf{z}_{\alpha}}(t)$ of the unperturbed differential system (25) associated to the $2 \pi$-periodic solution

$$
\left(\begin{array}{c}
Z(t) \\
V(t) \\
X(t) \\
Y(t)
\end{array}\right)=\left(\begin{array}{c}
Z_{0} \cos t-V_{0} \sin t \\
V_{0} \cos t+Z_{0} \sin t \\
0 \\
0
\end{array}\right)
$$

such that $M_{\mathbf{z}_{\alpha}}(0)$ be the identity of $\mathbb{R}^{4}$, we get

$$
M(t)=M_{\mathbf{z}_{\alpha}}(t)=\left(\begin{array}{cccc}
\cos t & -\sin t & t \cos t & -t \sin t \\
\sin t & \cos t & t \sin t & t \cos t \\
0 & 0 & \cos t & -\sin t \\
0 & 0 & \sin t & \cos t
\end{array}\right) .
$$

Note that the matrix $M_{\mathbf{z}_{\alpha}}(t)$ does not depend of the particular orbit periodic $\mathbf{x}(t)$. The inverse of the matrix $M(t)$, is given by

$$
M^{-1}(t)=\left(\begin{array}{cccc}
\cos t & \sin t & -t \cos t & -t \sin t \\
-\sin t & \cos t & t \sin t & -t \cos t \\
0 & 0 & \cos t & \sin t \\
0 & 0 & -\sin t & \cos t
\end{array}\right)
$$

Therefore the matrix

$$
M^{-1}(0)-M^{-1}(2 \pi)=\left(\begin{array}{cccc}
0 & 0 & -2 \pi & 0 \\
0 & 0 & 0 & -2 \pi \\
0 & 0 & 0 & 0 \\
0 & 0 & 0 & 0
\end{array}\right)
$$

satisfies the conditions of Theorem 7 . So we can apply it to system (25). Now $\xi^{\perp}(Z, V, X, Y)=(Z, V)$. We calculate the function $\mathcal{F}(\alpha)=\mathcal{F}\left(Z_{0}, V_{0}\right)$, where $\mathcal{F}(\alpha)$ is defined in (11). We find that

$$
\mathcal{F}(\alpha)=\left(\begin{array}{c}
-\frac{1}{2 \pi} \int_{0}^{2 \pi} F(t, a, b, c, d) \sin t d t \\
-\frac{1}{2 \pi} \int_{0}^{2 \pi} F(t, a, b, c, d) \cos t d t
\end{array}\right),
$$


where $a, b, c$ and $d$ are given in (5). Then, by Theorem 7 we have that for every simple zero $\left(Z_{0}^{*}, V_{0}^{*}\right) \in V$ of the function $\mathcal{F}(\alpha)$ we have a periodic solution of system (25) such that

$$
\left(\begin{array}{c}
Z(t) \\
V(t) \\
X(t) \\
Y(t)
\end{array}\right)=\left(\begin{array}{c}
Z_{0} \cos t-V_{0} \sin t \\
V_{0} \cos t+Z_{0} \sin t \\
0 \\
0
\end{array}\right), \quad \text { as } \varepsilon \rightarrow 0
$$

Going back through the changes of coordinates we get a periodic solution $(x, y, z, v)(t)$ of system (21) such that

$$
x(t, \varepsilon) \rightarrow \frac{1}{2}\left(V_{0}^{*} \sin t-Z_{0}^{*} \cos t\right) \quad \text { as } \quad \varepsilon \rightarrow 0 .
$$

Of course,it is easy to check that the previous expression provides a periodic solution of the linear differential equation $\dddot{x}+2 \ddot{x}+x=0$. Hence the theorem is proved.

\section{Proof of Corollary 5}

Proof of Corollary 5. If $F(t, x, \dot{x}, \ddot{x}, \dddot{x})=\sin x+b \sin t$, We show that

$$
\mathcal{F}\left(r_{0}\right)=J_{1}\left(\frac{r_{0}}{2}\right),
$$

where $J_{1}(z)$ is the Bessel function of first kind. This function has infinitely many simple zeros when $r_{0} \rightarrow \infty$, see for more details [1]. In this case the differential system has as many periodic orbits as we want taking $\varepsilon$ sufficiently small. Hence the corollary is proved.

\section{ACKNOWLEDGEMENTS}

The first author is partially supported by a MEC/FEDER grant MTM200803437, by a CIRIT grant number 2009SGR-410 and by ICREA Academia.

\section{REFERENCES}

[1] M. Abramowitz and I.A. Stegun, Bessel Functions $J$ and $Y, \S 9.1$ in Handbook of Mathematical Functions with Formulas, Graphs, and Mathematical Tables, 9th printing. New York: Dover, pp. 358-364, 1972.

[2] G.J.B. Van den Berg, L.A. Peletier and W.C. Troy, Global branches of multi-bump periodic solutions of the Swift-Hohenberg equation, Arch. Ration. Mech. Anal. 158 (2001), $91-153$.

[3] A. Buică, J. P. Françoise and J. Llibre, Periodic solutions of nonlinear periodic differential systems with a small parameter, Comm. on Pure and Appl. Anal. 6 (2007), 103-111.

[4] B. Buffoni, M. Groves and J.F. Toland, A plethora of capillary-gravity waves with nearcritical Bond and Froude numbers, Philos. Trans. Roy. Soc. London Ser. A 354 (1996), $575-607$.

[5] A.V. BuRYAK AND N.N. AkHmediev, Stability criterion for stationary bound states of solitons with radiationless oscillating tails, Phys. Rev. E 51 (1995), 3572-3578.

[6] J.G.B. ByатT-Sмith, On the change of amplitude of interacting solitary waves, J. Fluid Mech. 182 (1987), 467-483.

[7] P.C. Carrião, L.F.O. Faria and O:H. Miyagaki, Periodic solutions for extended FisherKolmogorov and Swift-Hohenberg equations by truncature techniques, Nonlinear Anal. 67 (2007), 3076-3083.

[8] A.R. Champneys, Homoclinic orbits in reversible systems and their applications in mechanics, fluids and optics, Physica D 112 (1998), 158-186.

[9] A.R. Champneys and M.D. Groves, A global investigation of solitary wave solutions to a two-parameter model for water waves, J. Fluid Mech. 342 (1997), 199-229. 
[10] W. Craig AND M.D. GRoves, Hamiltonian long-wave approximations to the water-wave problem, Wave Motion 19 (1994), 367-389.

[11] J. GuCKenheimer And P. Holmes, Nonlinear oscillations, dynamical systems and bifurcations of vectors fields, Springer, 1983.

[12] G.W. Hunt, H.M. Bolt And J.M.T. Thompson, Structural localisation phenomena and the dynamical phase-space analogy, Proc. Roy. Soc. London A 425 (1989), 245-267.

[13] G.W. Hunt AND M.K. WadeE, Comparative Lagrangian formulations for localised buckling, Proc. Roy Soc. London A 434 (1991), 485-502.

[14] J.K. Hunter AND J. Scheurle, Existence of perturbed solitary wave solutions to a model equation for water-waves, Physica D 32 (1988), 253-268.

[15] M. KARLSSON AND A. HÖÖK, Soliton-like pulses governed by fourth order dispersion in optical fibres, Opt. Com. 104 (1994), 303-307.

[16] S. KichenASSAmy, Existence of solitary waves for water-wave models, Nonlinearity 10 (1997), $133-151$.

[17] S. Kichenassamy And P.J. Olver, Existence and non-existence of solitary wave solutions to higher-order model evolution equations, SIAM J. Math. Anal. 23 (1996), 1141-1166.

[18] S. LANG, Algebra, 3rd.edition, Addison-Wesley, 1993.

[19] J. Llibre AND A. MAKhlouf, On some periodic orbits of the fourth-order differential equation $u^{\prime \prime \prime \prime}+q u^{\prime \prime}+p u=\varepsilon F\left(u, u^{\prime}, u^{\prime \prime}, u^{\prime \prime \prime}\right)$, submitted, 2010.

[20] J. Llibre, S. Rebollo-Perdomo And J. Torregrosa, Limit cycles bifurcating from isochronous surfaces in $\mathbb{R}^{3}$, J. Math. Anal. and Appl. 381 (2011), 414-426.

[21] J. LliBRE AND M.A. TEIXEIRA, On the periodic orbits of a fourth-order differential equation, J. Math. Anal. and Appl. 387 (2012), 181-188.

[22] I.G. MALkin, Some problems of the theory of nonlinear oscillations, (Russian) Gosudarstv. Izdat. Tehn.-Teor. Lit., Moscow, 1956.

[23] T.R. Marchant and N.E Smyth, Soliton interaction for the extended Korteweg-le Vries equation, IMA J. Appl. Math. 556 (1996), 157-176.

[24] P. Olver, Classical invariant theory, London Math. Soc. Student Texts, vol. 44, Cambridge Univ. Press, New York, 1999.

[25] L.A. Ostrovsky, K.A. Gorshkov, V.V. Papko and A.S. Pikovsky, On the existence of stationary solitons, Phys. Lett. A 74 (1979), 177-179.

[26] L.A. Peletier And W.C. Troy, Spatial Patterns, Higher Order Models in Physics and Mechanics, Brikhäuser, 2001.

[27] M. Roseau, Vibrations non linéaires et théorie de la stabilité, (French) Springer Tracts in Natural Philosophy, Vol.8 Springer-Verlag, Berlin-New York, 1966.

[28] J. Sanders And F. Vehrulst, Averaging method in nonlinear dynamical systems, Applied Mathematical Sciences. 59, Springer, 1985.

[29] F. Vehrulst, Nonlinear differential equations and dynamical systems, Universitext, Springer 1996.

[30] J.A. ZuFIRIA, Symmetry breaking in periodic and solitary gravity-capillary waves on water of finite depth, J. Fluid Mech. 184 (1987), 183-206.

1 Departament de Matematiques, Universitat Autònoma de Barcelona, 08193 Bellaterra, Barcelona, Catalonia, Spain

E-mail address: jllibre@mat.uab.cat

2 Department of Mathematics, University of Annaba, BP 12, Annaba, Algeria

E-mail address: makhloufamar@yahoo.fr 